\title{
Comment? 4000 francs pour un titre postgrade fédéral?
}

\author{
Pourquoi le titre de spécialiste ne coûtera finalement que 2000 francs pour les membres de la FMH - \\ Informations et considérations sur les nouveaux tarifs
}

Ch. Hänggeli, responsable du secrétariat pour la formation prégraduée, postgraduée et continue (FPPC)

En vertu de la nouvelle réglementation fédérale, les titres de spécialiste ne seront plus attribués exclusivement aux membres de la FMH. En effet, les titres FMH peuvent désormais être décernés aux non-membres. Les frais supportés par la FMH dans le domaine de la formation postgraduée et continue ne pourront donc plus être financés uniquement par les cotisations de membres. Dès lors, il s'agira de couvrir les frais par le biais des émoluments perçus sur les diplômes. Conformément au nouveau tarif décidé par la Chambre médicale et par le Comité central, le premier titre de spécialiste reviendra à 4000 francs. Cependant, les membres de la FMH pourront profiter de la solidarité «intergénérations», car ils ne paieront que 2000 francs par titre grâce à l'utilisation d'une grande partie des cotisations de membres en faveur des jeunes médecins. spécialiste sera reconnu dans tous les Etats de l'UE. Les assistants en formation bénéficieront pour leur part de moyens de recours qui ne sont pas négligeables sur le chemin parfois semés d'embûches conduisant à l'obtention d'un titre de spécialiste. En outre, la suppression des exigences du doctorat et de l'affiliation à la FMH pour l'obtention d'un titre postgrade fédéral représente également une simplification de la procédure. Quant aux médecins praticiens sans titre de spécialiste, la nouvelle loi leur offre aussi de nouvelles possibilités d'obtenir a posteriori un titre de spécialiste à des conditions facilitées [2].

\section{Et qu'en est-il des tarifs?} sonnes et la loi fédérale sur l'exercice des professions médicales (LEPM) [1] sont entrés simultanément en vigueur le $1^{\text {er }}$ juin 2002, donnant une nouvelle base légale à la formation postgraduée des médecins en Suisse. Ainsi donc, les titres de spécialiste FMH ne seront bientôt plus que de l'histoire ancienne. Ils ont été remplacés par 43 nouveaux titres fédéraux de spécialiste et par celui de «médecin praticien». Mais si l'emballage est modifié, le contenu reste le même. En effet, pour les assistants concernés, les exigences de formation postgraduée ne changent pas, car la Confédération reconnaît la Réglementation pour la formation postgraduée de la FMH et tous les programmes de formation y afférents, confiant à cette dernière la légitimation officielle à laquelle elle aspirait depuis longtemps déjà.

\section{Qu'est-ce qui change?}

La nouvelle présentation des diplômes de formation postgrade, signés par le président de la FMH et le directeur de l'Office fédéral de la santé publique n'est pas la seule innovation - et de loin pas la plus importante - apportée par la LEPM (cf. tableau 1). Bien plus significatif est le fait que l'ensemble des médecins de Suisse pourront circuler librement en Europe et que leur titre de de francs dans le domaine de la formation postgraduée et continue, soit une part importante de son budget. Les émoluments de diplôme s'élevaient jusqu'ici à 800 francs par titre pour quelque 800 octrois par année. Il est bien clair que les frais de la FMH ne sont ainsi pas couverts. La FMH n'a jamais touché et ne touchera malheureusement jamais aucun denier public pour ses tâches dans le domaine de la formation postgraduée et continue. Dès lors, la majeure partie des frais doit être supportée par les cotisations des membres. A cet égard, les quelque 30000 membres de la FMH ont toujours soutenu ce domaine de manière déterminante, au sens d'une solidarité entre générations.

La suppression de l'affiliation obligatoire pour l'obtention et le port d'un titre fédéral de spécialiste implique que les frais inhérents au domaine de la formation postgraduée et continue soient couverts par des émoluments. Car il va de soi qu'on ne peut pas demander aux membres de la FMH de financer la formation de nonmembres par leurs cotisations. Le Comité central s'est donc vu contraint d'adapter les tarifs à la nouvelle donne. Résultat: le titre fédéral de spécialiste reviendra désormais à 4000 francs.
La FMH injecte annuellement quelque 4 millions 
Tableau 1

Les innovations en un coup d'œil.

- Titres postgrades fédéraux et donc reconnus au sein de I'UE;
- libre circulation des personnes dans les pays de I'UE;
- obtention d'un titre postgrade fédéral en tant que condition essentielle pour l'ouverture d'un
cabinet médical;
- voies de droit étendues pour les médecins en formation postgraduée;
- suppression de l'obligation d'adhérer à la FMH pour l'obtention d'un titre postgrade fédéral;
- suppression du doctorat comme condition à l'obtention d'un titre postgrade;
- émoluments couvrant les frais de diplôme;
- dispositions transitoires légales facilitant l'obtention d'un titre postgrade fédéral aux praticiens
sans titre de spécialiste;
- formation continue obligatoire pour tous les détenteurs d'un titre postgrade;
- prescriptions nationales concernant les désignations professionnelles.

\section{Solidarité «intergénérations» entre les membres de la FMH}

Il est évident que 4000 francs, c'est cher. C'est pourquoi lors de la discussion sur le budget en mai 2001, la Chambre médicale a adhéré à la proposition visant à maintenir une solidarité «intergénérations» entre les membres de la FMH et à créer une incitation à s'affilier à la FMH en récompensant les membres FMH de leur fidélité par la rétrocession d'une partie de leurs cotisations lors de l'obtention de leur premier titre de spécialiste et par une réduction de leurs cotisations pour les 5 années suivantes! Ainsi, le titre de spécialiste ne coûtera en fait plus que 2000 francs pour les membres de la FMH.

La Chambre médicale est même allée plus loin dans la rétrocession et la réduction future des cotisations, qui seront bien entendu à la charge des comptes généraux de la FMH. L'organe suprême du corps médical a également prévu un montant annuel important au budget général de la FMH en faveur de projets pour les médecins en formation postgraduée. La gestion du secrétariat FPPC en tant que centre de charges garantit une séparation claire des dépenses et des recettes de ce domaine dans les comptes généraux et la transparence de son financement vis-à-vis de la Confédération et du public.

\section{II vaut la peine de s'affilier à la FMH!}

Les changements décrits concernant les cotisations démontrent qu'il est profitable pour les médecins d'adhérer à la FMH dès leur première année de formation postgraduée. De plus, les cotisations sont un bon investissement, dans la mesure où tous les renseignements ayant trait à la formation postgraduée (allant de la simple demande à l'élaboration d'un plan de formation, etc.) sont totalement gratuits pour les membres de la FMH!

\section{0 francs ou 800 francs, la date limite est déterminante}

Un montant de 4000 francs pour le premier titre de spécialiste, ce n'est pas rien surtout si l'on songe à la pression exercée sur les salaires des assistants suite à la diminution de l'horaire de travail et aux frais qui, selon la discipline ou l'hôpital, peuvent être déjà très élevés durant la formation postgraduée (cours de médecine d'urgence et autres, obtention de certificats d'aptitude technique, examen de spécialiste, etc.). Le fait qu'en tant que membre de la FMH, la plupart des porteurs de titre de spécialiste bénéficient des rétrocessions et des réductions de cotisations est malgré tout une consolation. Plus chanceux sont les candidats qui ont terminé leur formation postgraduée avant le $1^{\text {er }}$ juin 2002, soit avant l'entrée en vigueur de la LEPM, car ils obtiendront le nouveau titre fédéral de spécialiste encore pour 800 francs. Vous trouverez de plus amples détails concernant les dispositions transitoires et d'introduction dans le nouveau tarif ci-après.

\section{Et qu'offre-t-on pour ce prix?}

En premier lieu un diplôme de spécialiste autorisant son détenteur à exercer la médecine de manière indépendante en Suisse et dans toute l'Europe. Mais bien d'autres choses encore. Ce n'est en effet pas seulement la paperasserie accompagnant la demande d'un titre de spécialiste qui est très coûteuse. Les coûts élevés sont produits pour la plupart en amont par l'élaboration et la révision des programmes de formation et leur application. Ils exigent en effet le concours d'un grand nombre de spécialistes et de commissions. A cet égard, il n'y a qu'à penser à la Commission des titres, qui traite chaque année quelque 2000 demandes de renseignements, plans de formation et demandes de titres, ou à la Commission des établissements de formation postgraduée, chargée de la reconnaissance et de la classification d'environ 1500 hôpitaux. 


\section{Perspectives}

La FMH, en tant qu'organisation accréditée par la Confédération, est responsable de la qualité de la formation postgraduée et continue des médecins. Or, les programmes de formation à eux seuls ne suffisent pas à garantir la qualité souhaitée. Malgré de louables efforts de la part de nombreux responsables d'établissements de formation reconnus, on constate encore des lacunes. La formation postgraduée laisse souvent à désirer tant sur le plan structurel que théorique, les objectifs de formation fixés dans les programmes de formation ne peuvent pas être entièrement atteints, la part «prestations» étant si importante dans les postes d'assistants que la formation reste le parent pauvre. Il convient donc d'agir sans tarder dans ce domaine. Divers projets d'amélioration de la qualité de la formation postgraduée sont en cours ou pour certains déjà réalisés, à savoir:

- l'évaluation des établissements de formation par les assistants;

- l'établissement de concepts de formation postgraduée;
- la visite des établissements de formation dans le cadre de leur reconnaissance;

- l'encouragement de l'assistanat au cabinet médical;

- la vérification des objectifs de formation en termes de faisabilité;

- l'introduction de cours de management.

Le nouveau tarif et la contribution de solidarité prélevée sur les cotisations des membres constituent les bases indispensables pour atteindre le but visé, à savoir une sensible amélioration de la qualité de la formation postgraduée en collaboration avec toutes les personnes et institutions concernées.

\section{Références}

1 Loi fédérale concernant l'exercice des professions médicales dans la Confédération suisse.

2 Hänggeli C. Titres de spécialiste pour tous? Octroi de titres fédéraux de formation postgrade à des médecins sans titre en vertu des dispositions transitoires. Bull Méd Suisses 2002;83(25):1284-8.

\section{Tarif}

Diplômes et autres prestations de services du Secrétariat pour la formation prégraduée, postgraduée et continue (FPPC)

\section{Titre postgrade fédéral}

Premier titre de spécialiste

Fr. 4000.-

Exclusivement pour membres de la FMH

- rétrocession sur la cotisation de membre: jusqu'à Fr. 200.- par année d'affiliation durant 5 ans au maximum

- réduction de la cotisation de membre: jusqu'à Fr. 200.- par année au maximum pendant 5 ans

Deuxième titre de spécialiste

Chaque titre de spécialiste suivant

«médecin praticien»

Fr. 2000.-

(pris en compte lors de l'obtention d'un titre de spécialiste)

\section{Titres FMH (décernés exclusivement aux membres de la FMH)}




\section{Prestations de services}

Temps consacré

Renseignements écrits par le Secrétariat FPPC

30 min. à 1 heure

1 à 2 heures

plus de 2 heures

Etablissement d'un plan de formation postgraduée par la Commission des titres

30 min. à 1 heure

1 à 2 heures

plus de 2 heures

Etablissement d'une attestation d'équivalence (uniquement pour les porteurs d'un diplôme de médecin non reconnu)

Etablissement d'un duplicata en cas de perte de diplôme

\section{Membres Non-membres}

gratuit

Fr. 100.-

gratuit

Fr. 200.-

gratuit

Fr. 300.-

gratuit

Fr. 100.-

gratuit

Fr. 200.-

gratuit

Fr. 300.-

Fr. 1000.-

Fr. 150.-

\section{Remarques}

- Pour les titres de formation postgrade, la facture dressée après réception de la demande, est payable dans les 30 jours. Le diplôme n'est remis qu'après réception du versement.

- Les émoluments pour l'examen de spécialiste sont fixés dans le règlement d'examen de chaque société de discipline médicale.

- Les émoluments déjà versés pour le titre postgrade de «médecin praticien» ou pour l'attestation d'équivalence sont pris en compte lors de l'octroi d'un titre de spécialiste.

\section{Dispositions transitoires et complémentaires sur les tarifs}

- Les médecins-assistants qui termineront leur formation postgraduée d'ici au $1^{\text {er }}$ juin 2002 (nombre d'années de stages et structure selon programme) obtiendront le titre fédéral de spécialiste encore au prix de Fr. 800.-, selon l'ancien tarif, cela pour autant qu'ils remplissent toutes les autres conditions pour l'obtention du titre d'ici à fin 2002 au plus tard (cours, examen de spécialiste, travail scientifique, etc.) et que tous les documents exigés soient parvenus à la Commission des titres d'ici au 31 décembre 2002. Il en va de même pour le titre postgrade fédéral de «médecin praticien» (Fr. 250.-).

- Les candidats dont la demande de titre a fait l'objet d'une décision définitive après le $1^{\text {er }}$ mai 2002, reçoivent déjà le titre postgrade fédéral correspondant.
- Les candidats ayant obtenu un titre de spécialiste FMH ou un «certificat LAMal» avant le $1^{\text {er }}$ juin 2002 peuvent obtenir le diplôme fédéral correspondant en tant que duplicata au prix de Fr. 150.-. Attention: cette démarche est facultative, la loi ne prescrit rien en la matière. Le titre de spécialiste FMH étant absolument équivalent au titre fédéral de spécialiste, les médecins qui ont exercé jusqu'ici sans titre de spécialiste pourront continuer à le faire sans titre fédéral de formation postgrade!

- Les médecins qui, en vertu des dispositions transitoires de l'ordonnance à la LEPM, ont droit à un titre fédéral de spécialiste devront s'acquitter de Fr. 4000.- (pour les membres de la FMH naturellement sous réserve de rétrocessions et de réductions éventuelles des cotisations de membres).

- Ces dispositions sont valables également pour les formations approfondies. Cas particulier: les formations approfondies qui, en vertu des dispositions transitoires du programme de formation postgraduée, peuvent être attribuées sans autre aux détenteurs des titres de spécialiste concernés (chirurgie ophtalmologique, urologie chirurgicale) ne coûtent que Fr. 200.-.

Approuvé par le Comité central (CC) de la FMH le 24 avril 2002. 